\title{
Extended Straightforward Current Control for Permanent Magnet Synchronous Machines
}

\author{
Andreas Liske, Philipp Hofmeier, Michael Braun \\ Elektrotechnisches Institut (ETI) - Electrical Drives and Power Electronics \\ Kaiserstr. 12 \\ 76131 Karlsruhe, Germany \\ Phone: +49 (0) 721-608-46848 \\ Fax: +49 (0) 721-358854 \\ Email: Andreas.Liske@kit.edu \\ URL: http://www.eti.kit.edu
}

\begin{abstract}
Keywords
$<<$ Adaptive Control $>>,<<$ Highly Dynamic Drive $>>,<<$ Permanent Magnet Motor $>>,<<$ Optimal Control $>>,<<$ Sensorless Control $>>$.
\end{abstract}

\begin{abstract}
This paper presents a direct, adaptive and parameter-free current control scheme for synchronous machines with excellent control quality and high dynamics. For magnetic anisotropic synchronous machines $\left(L_{d} \neq L_{q}\right)$, it automatically allows easy identification of the rotor position angle in the whole speed range, making it particularly suitable for sensorless control.
\end{abstract}

\section{Introduction}

Since many years field oriented control (FOC) is known as one of the most common control schemes for three phase motors. Besides that, many predictive control strategies were investigated and published during the last years, with the goal to allow an ideal setting of the control parameters, increase robustness, replace the mechanical speed and torque sensors or simply to reduce the time and effort during initial implementation of a new drive system setup [1]. The drawbacks of those control schemes are versatile and often combined. One of them is the dependency on machine parameters that may vary during operation, leading to a suboptimal control setup. Another is the use of complex mathematical machine models to calculate and correct the machine's parameters or the system behavior, which increases the required computing time and collides with the hard real time demands of a high-dynamic drive control system. If hysteresis controllers are used, then the desired setpoint value never is reached accurately, because the switching frequency is limited by the maximum switching losses and in addition to that, the varying switching frequency makes appropriate filter design a complex task. Notably, the model predictive control (MPC) [2] and the field of sensorless control [3] has been investigated exhaustively, so that meanwhile there are improvements and advanced control strategies available, that have overcome the drawbacks for some of those control schemes $[4,5]$.

In $[6,7,8]$ the "Straightforward Current Control" (SCC) scheme has been presented for the control of a DC machine as well as for the control of magnetic isotropic 3-phase synchronous machines (with $L_{d}=L_{q}$ ), that delivers excellent control quality and high dynamics. At the same time it doesn't need any machine parameters, no machine model, no test pulses, no offline calculations or cost functions and the computational effort is comparatively little. The system is identified permanently in every pulse period by measuring the slopes of the stator currents in each of the applied switching states, making the SCC completely adaptive.

In this paper an extended algorithm of this straightforward current control strategy is presented (in the following called Extended Straightforward Current Control - ESCC), that additionally allows to control 


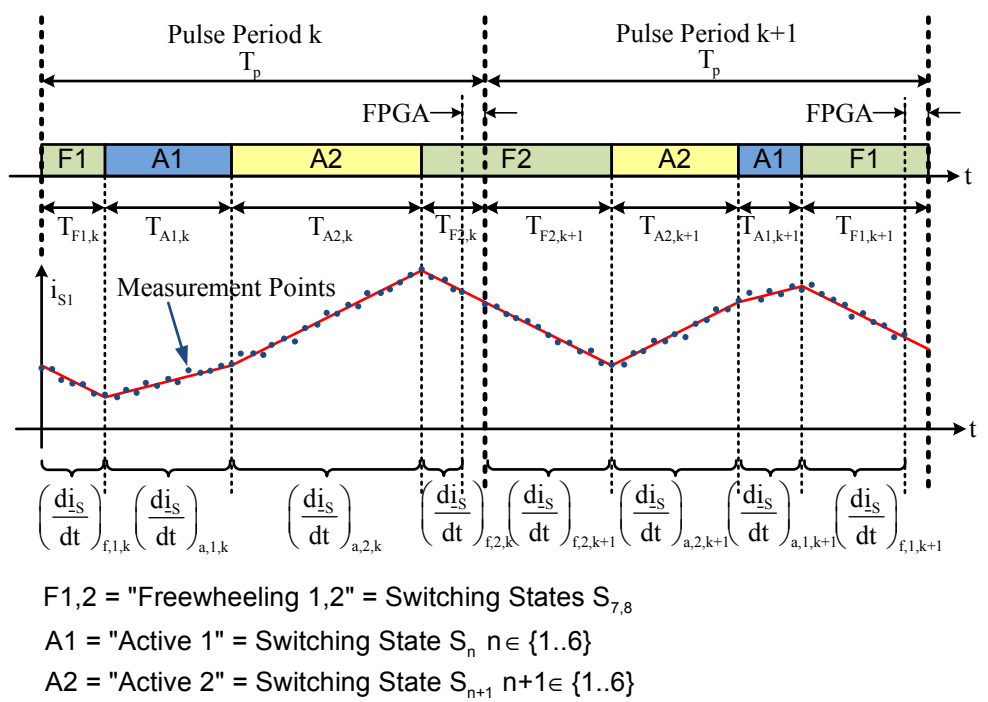

Figure 1: Timing, modulation and measurement principle of the SCC and ESCC. Although all three stator phase currents are measured, in this diagram only $i_{S 1}$ is outlined to improve clarity.

the current of synchronous machines with magnetic anisotropic characteristics $\left(L_{d} \neq L_{q}\right)$ like e.g. interior permanent magnet synchronous machines (IPMSM), as well as magnetic isotropic machines $\left(L_{d}=L_{q}\right)$ without changes.

The ESCC algorithm can easily be used to identify the rotor position angle for magnetic anisotropic machines in the whole speed range, including low speed and standstill at no extra cost. This makes this control scheme particularly suitable for sensorless control.

Before the ESCC is presented, a short summary of the SCC is given in the first section of this paper. This is done to explain the common basic working principle of both control schemes, and because with a direct comparison, the additional benefits of the ESCC can be shown.

\section{Basic Straightforward Current Control scheme (SCC)}

The basis for the SCC scheme $[6,7,8]$ and hence for the ESCC scheme presented in this paper is the fast detection of the stator current slopes during each switching state $S_{n}$ with $n \in\{1 . .8\}$ of the utilized voltage source inverter (VSI). This is done by measuring the stator currents during each switching state in every pulse period very fast with oversampling of the A/D-converter and calculating the current slopes with a least-squares-estimator-algorithm to eliminate noise at the end of the same period (index k) in an FPGA (see figure1). Due to the fast calculation possibilities in the FPGA, the results can be utilized directly for the calculation of the duty-cycles of the next pulse period $\mathrm{k}+1$, which minimizes control dead-time significantly. The switching frequency is assumed to be high enough so that the current slopes can be considered as being linear during the switching states.

The so gained current slopes $\left(\frac{d i_{S}}{d t}\right)_{a, n, k}$ of pulse period $\mathrm{k}$ with the index "a", for the six active switching states $S_{n}$ with $n \in\{1 . .6\}$, where the machine is connected to the dc link voltage, and $\left(\frac{d i_{S}}{d t}\right)_{f, k}$ for the two freewheeling switching states $S_{7,8}$ (index "f"), where all switches of the VSI are connected to either the positve or the negative dc link terminal, are used to define the characteristic values $\Delta \underline{i}_{a, n, k}$ and $\underline{i}_{f, k}$ :

$$
\begin{aligned}
\Delta \underline{i}_{a, n, k} & \left.=\left[\left(\frac{\mathrm{d}}{\mathrm{dt}^{i}}\right)_{S}\right)_{a, n, k}-\left(\frac{\mathrm{d}}{\mathrm{dt}^{2}} i_{S}\right)_{f, k}\right] \cdot T_{p} \\
\Delta \underline{i}_{f, k} & =\left(\frac{\mathrm{d}}{\mathrm{dt}^{-} \underline{i}_{S}}\right)_{f, k} \cdot T_{p}
\end{aligned}
$$

An illustration of those characteristic values is displayed in figure 2(a). The characteristic values $\Delta \underline{i}_{a, n, k}$ describe the current variation that would occur, if only the voltage corresponding to the active switching 


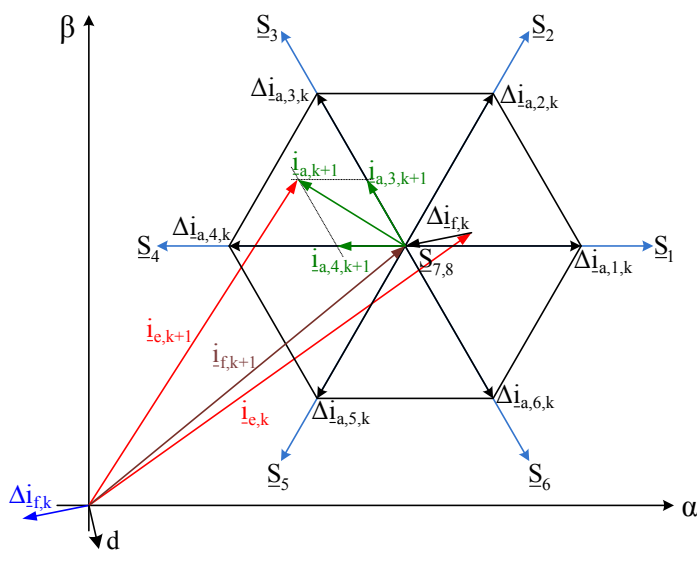

(a) Synchronous machines with $L_{d}=L_{q}$

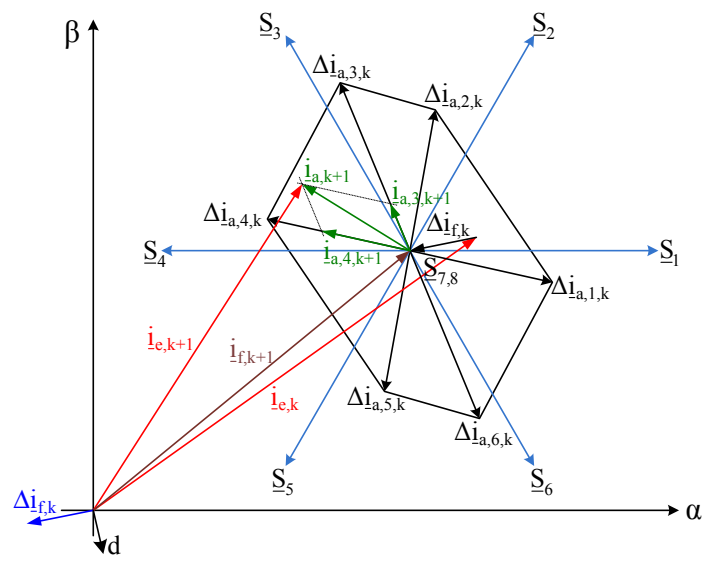

(b) Synchronous machines with $L_{d} \neq L_{q}$

Figure 2: Characteristic values $\Delta \underline{i}_{a, n, k}$ and $\Delta \underline{i}_{f, k}$ (black vectors), switching state vectors $\underline{S}_{n}$ (blue), area of possible current variation in one pulse period $\mathrm{k}$ and the vector diagram of the stator current variation during pulse period $\mathrm{k}+1$ for synchronous machines with $L_{d}=L_{q}$ and $L_{d} \neq L_{q}$

state with the index $\mathrm{n}$ would be applied to the machine for the whole pulse period $\left(T_{p}\right)$ with the index k. Similarly $\Delta \underline{i}_{f, k}$ depicts the current variation that would occur, if only a freewheeling switching state would be applied to the machine for the whole pulse period $\mathrm{k}$.

For synchronous machines with magnetic isotropic characteristics $\left(L_{d}=L_{q}\right)$, which is a basic presumption in the SCC scheme, all six active characteristic values $\Delta \underline{i}_{a, n, k}$ have the same length and the argument is the same as the one of the corresponding switching state vector $\underline{S}_{n}$ (see figure 2(a))[8]. So in the SCC scheme the measurement of only one active switching state is sufficient to know all six active characteristic values.

$$
\begin{aligned}
\left.\Delta \underline{i}_{a, n, k}\right|_{L_{d}=L_{q}}=\Delta I_{a, k} & =\frac{2}{3} \cdot \frac{U_{D C, k} T_{p}}{L_{k}} \cdot \underline{S}_{n} & & \\
\underline{S}_{n} & =e^{j \varphi_{n}} & & \\
\varphi_{n} & =(n-1) \cdot \frac{\pi}{3} & & \mathrm{n} \in\{1 . .6\}
\end{aligned}
$$

This spans an equilateral hexagon of the possible current variation, that can be reached within one pulse period, similar to the hexagon known from conventional space vector modulation (see fig.2(a)). Since the inner voltage of the machine is effective anyway during the whole pulse period, the origin of this hexagon is at the tip of the vector $\underline{i}_{f, k+1}$, which designates the point where the current space vector would be, if no active switching state would be applied during the pulse period $\mathrm{k}+1$. It can be calculated with the current space vector $\underline{i}_{e, k}$, which describes the last value of the stator current at the end of the preceding pulse period $\mathrm{k}$, by simply adding $\Delta \underline{i}_{f, k}$ :

$$
\underline{i}_{f, k+1}=\underline{i}_{e, k}+\Delta \underline{i}_{f, k}
$$

Since the control algorithm in the FPGA is started shortly before the end of the current pulse period k, the necessary value $\underline{i}_{e, k}$ for this equation can not be measured, but can be calculated by extrapolation of the just measured current slopes and the knowledge of the applied duty cycles in period $\mathrm{k}$.

The green vector diagram in figure 2 depicts the essential of the straightforward current control algorithm: The necessary duty cycles to reach the desired current setpoint value $\underline{i}_{e, k+1}$ at the end of period $\mathrm{k}+1$ can be obtained by the projection of the vector $\underline{i}_{a, k+1}$ to the adjacent switching state vectors $\underline{S}_{n}$. This is done by using the same computation formulas as with the well known space vector modulation.

$$
\underline{i}_{a, k+1}=\underline{i}_{e, k+1}-\underline{i}_{f, k+1}=\underline{i}_{e, k+1}-i_{e, k}-\Delta i_{f, k}
$$




\section{Extended Straightforward Current Control scheme (ESCC)}

\section{Generalized definition of the characteristic values}

One main limitation of the SCC control scheme is that it is only suited for magnetic isotropic machines with $L_{d}=L_{q}$. When it is applied to a magnetic anisotropic machine with $L_{d} \neq L_{q}$, the control quality decreases with a growing magnetic anisotropy. The reason for this is the assumption, that the six $\Delta \underline{i}_{a, n, k}$ are all of the same length and that they point in the same direction as the corresponding switching state vectors, which is correct for $L_{d}=L_{q}$ but not for $L_{d} \neq L_{q}$.

To be able to use the same basic control strategy of the straightforward current control scheme to control machines with magnetic anisotropic characteristics, a closer examination of the response of the stator currents to the applied switching states with respect to $L_{d} \neq L_{q}$ is necessary. Starting point are the system equations of the synchronous machine, transformed into the stator-oriented $\alpha \beta$-reference frame [9]:

$$
\left(\underline{\mathrm{u}}_{S}\right)=\left(R_{S}\right)\left(\underline{i}_{S}\right)+\left(\underline{L}_{A}\right) \cdot \frac{d}{d t}\left(\underline{i}_{S}\right)+\frac{d}{d t}\left(\underline{L}_{B}\right) \cdot\left(\underline{i}_{S}\right)+\left(\underline{L}_{B}\right) \cdot \frac{d}{d t}\left(\underline{i}_{S}\right)+\frac{d}{d t}\left(\underline{\Psi}_{P M S}\right)
$$

with

$$
\begin{aligned}
& \left(\underline{L}_{A}\right)=\left(\begin{array}{ccc}
\frac{3}{2} L_{A} & 0 & 0 \\
0 & \frac{3}{2} L_{A} & 0 \\
0 & 0 & 0
\end{array}\right) \\
& \left(\underline{L}_{B}\right)=\frac{3}{2} L_{B} \cdot\left(\begin{array}{ccc}
\cos (2 \gamma(t)) & \sin (2 \gamma(t)) & 0 \\
\sin (2 \gamma(t)) & -\cos (2 \gamma(t)) & 0 \\
0 & 0 & 0
\end{array}\right) \\
& \mathrm{L}_{A}=\frac{1}{3}\left(L_{d}+L_{q}\right)
\end{aligned}
$$

With $L_{d}=L_{q}$, the terms with $\left(\underline{L}_{B}\right)$ are zero, which simplifies eq. 8 significantly and leads to the results that are used in the basic SCC scheme. For magnetic anisotropic machines all terms have to be considered. Equation 8 can be rewritten as a space vector, consisting of the two components $u_{S, \alpha}$ and $u_{S, \beta}$ :

$$
\begin{aligned}
\left(\underline{u}_{S}\right)=\left(\begin{array}{c}
u_{S, \alpha} \\
u_{S, \beta}
\end{array}\right)= & R_{S}\left(\begin{array}{c}
i_{S, \alpha} \\
i_{S, \beta}
\end{array}\right)+\left(\underline{L}_{A}\right)\left(\begin{array}{c}
\frac{d}{d t} i_{S, \alpha} \\
\frac{d}{d t} i_{S, \beta}
\end{array}\right) \\
& +\frac{d}{d t}\left(\underline{L}_{B}\right)\left(\begin{array}{c}
i_{S, \alpha} \\
i_{S, \beta}
\end{array}\right)+\left(\underline{L}_{B}\right)\left(\begin{array}{c}
\frac{d}{d t} i_{S, \alpha} \\
\frac{d}{d t} i_{S, \beta}
\end{array}\right)+\frac{d}{d t}\left(\underline{\Psi}_{P M S}\right)
\end{aligned}
$$

The equations of $u_{S, \alpha}$ and $u_{S, \beta}$ are solved for the derivatives of the current vector components $\frac{d}{d t} i_{S, \alpha}$ and $\frac{d}{d t} i_{S, \beta}$. With those, the characteristic value for the freewheeling switching states $\Delta \underline{i}_{f}$ can be calculated (see also eq. 2).

$$
\Delta \underline{i}_{f}=\left(\frac{\mathrm{d}}{\mathrm{dt}^{i}} i_{S}\right)_{f, k} \cdot T_{p}=\left.\left(\begin{array}{c}
\frac{d}{d t} i_{S, \alpha} \\
\frac{d}{d t} i_{S, \beta}
\end{array}\right)\right|_{\mathrm{u}_{S \alpha}=\mathrm{u}_{\mathrm{S} \beta}=0} \cdot T_{p}=\left(\begin{array}{c}
\Delta i_{f, \alpha} \\
\Delta i_{f, \beta}
\end{array}\right)
$$

with

$$
\begin{aligned}
\Delta i_{f, \alpha}=\frac{2 \cdot T_{p}}{3\left(L_{A}^{2}-L_{B}^{2}\right)} \cdot[ & \left(-R_{S} L_{A}+R_{S} L_{B} \cos (2 \gamma(t))+3 \omega L_{A} L_{B} \sin (2 \gamma(t))\right) \cdot i_{S, \alpha} \\
+ & \left(3 \omega L_{B}^{2}-3 \omega L_{A} L_{B} \cos (2 \gamma(t))+R_{S} L_{B} \sin (2 \gamma(t))\right) \cdot i_{S, \beta} \\
& \left.+\left(L_{A}+L_{B}\right) \Psi_{P M} \cdot \omega \cdot \sin (\gamma(t))\right] \\
\Delta i_{f, \beta}=\frac{2 \cdot T_{p}}{3\left(L_{A}^{2}-L_{B}^{2}\right)} \cdot[ & \left(-3 \omega L_{B}^{2}-3 \omega L_{A} L_{B} \cos (2 \gamma(t))+R_{S} L_{B} \sin (2 \gamma(t))\right) \cdot i_{S, \alpha} \\
+ & \left(-R_{S} L_{A}-R_{S} L_{B} \cos (2 \gamma(t))-3 \omega L_{A} L_{B} \sin (2 \gamma(t))\right) \cdot i_{S, \beta} \\
& \left.-\left(L_{A}+L_{B}\right) \Psi_{P M} \cdot \omega \cdot \cos (\gamma(t))\right]
\end{aligned}
$$




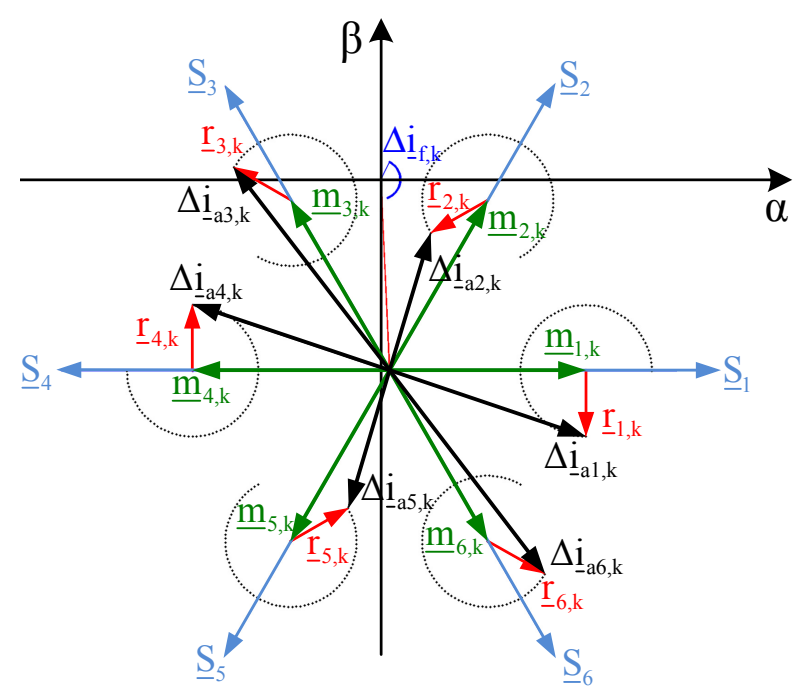

Figure 3: Geometrical locus of $\Delta \underline{i}_{a, n}$ for machines with $L_{d} \neq L_{q}$ after a $3 / 8$ electrical period and the vectors $\underline{m}_{n, k}$, $\underline{r}_{n, k}$ and $\Delta \underline{i}_{a, n, k}$ for the current pulse period $\mathrm{k}$.

The characteristic values of the six active switching states are (see also eq. 1)

$$
\Delta \underline{i}_{a, n}=\left(\begin{array}{c}
\frac{d}{d t} i_{S, \alpha} \\
\frac{d}{d t} i_{S, \beta}
\end{array}\right) \cdot T_{p}-\Delta \underline{i}_{f}=\left(\begin{array}{c}
\Delta i_{a, n, \alpha} \\
\Delta i_{a, n, \beta}
\end{array}\right)
$$

Solving and rearranging this equation leads to the following mathematical representation:

$$
\begin{aligned}
\Delta_{\underline{i}_{a, n}} & =\frac{2}{3\left(L_{A}^{2}-L_{B}^{2}\right)} \cdot u_{S} \cdot T_{p}\left(L_{A} e^{j \varphi_{n}}-L_{B} e^{j\left(2 \gamma(t)-\varphi_{n}\right)}\right) \\
& =\underbrace{\frac{2 u_{S} T_{p}}{3\left(L_{A}^{2}-L_{B}^{2}\right)} L_{A} e^{j \varphi_{n}}}_{\underline{m}_{n}}-\underbrace{\frac{2 u_{S} T_{p}}{3\left(L_{A}^{2}-L_{B}^{2}\right)} L_{B} e^{j\left(2 \gamma(t)-\varphi_{n}\right)}}_{\underline{r}_{n}} \\
& =\underline{m}_{n}-\underline{r}_{n}
\end{aligned}
$$

with

$$
\begin{aligned}
\underline{m}_{n, k} & =\frac{2 u_{S} T_{p}}{3\left(L_{A}^{2}-L_{B}^{2}\right)} \cdot L_{A} \cdot e^{j \varphi_{n}} \\
\underline{r}_{n, k} & =\frac{2 u_{S} T_{p}}{3\left(L_{A}^{2}-L_{B}^{2}\right)} \cdot L_{B} \cdot e^{j\left[2 \gamma_{k}-\varphi_{n}\right]} \\
u_{S} & =\left|\underline{u}_{S}\right|=\frac{2}{3} U_{D C} \\
\varphi_{n} & =(n-1) \cdot 60^{\circ} \quad \mathrm{n} \in\{1 . .6\}
\end{aligned}
$$

The six characteristic values for the current variation during the active switching states can each be represented by two vectors (fig.3). The constant, time-invariant vectors $\underline{m}_{n}$ point with the angle $\varphi_{n}$ in the direction of the corresponding switching state vectors $\underline{S}_{n}$. The time-variant vectors $\underline{r}_{n}$ all have the same constant length and rotate with the doubled angular frequency of the rotor position angle $2 \gamma(t)$ around the tip of $\underline{m}_{n}$, each with an individual angular offset of $-\varphi_{n}$. Because this angular offset sums up to $180^{\circ}$ when looking at two opposite switching states $S_{n}$ and $S_{n \pm 3}$, the characteristic values of opposite switching states show a symmetry with respect to the tip of $\underline{i}_{f, k+1}$ (see fig.2(b) and fig.3).

In Figure 3, the geometrical locus of the six $\Delta i_{a, n}$ during a three eighth electrical period is displayed. The length of $\underline{r}_{n}$ and with that the diameter of the black dotted circles directly depends on the difference between $L_{d}$ and $L_{q}$ (see also eq. 11,16,18) 


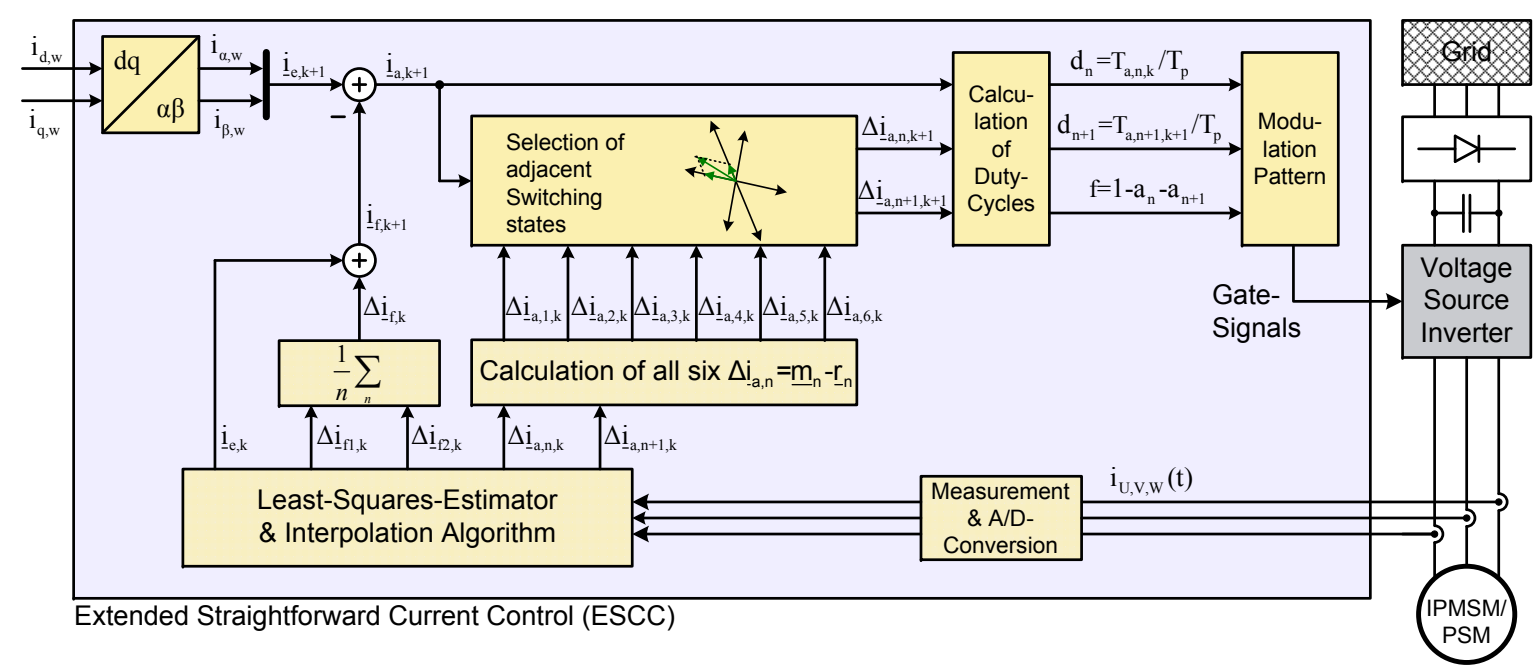

Figure 4: Block diagram of the presented extended straightforward current control scheme. The overlaying control loops, that deliver the required $i_{d, w}$ and $i_{q, w}$ are not displayed.

The meaning of those characteristic values is just the same as described for the basic SCC. The six $\Delta i_{a, n}$ describe the current variation, that would occur if only their corresponding switching state $S_{n}$ would be applied to the machine for the whole pulse period. In contrary to the SCC with $L_{d}=L_{q}$, the current response now varies in length and direction, depending on the switching state that is applied (fig.2(b)). For $L_{d}=L_{q}$, the equations 16 and 14 for the characteristic values are conform to the equations of the SCC scheme.

With this analysis of the stator current response to the switching states and the generalized definition of the characteristic values in equation 16, the extended straightforward current control scheme can be implemented for three-phase synchronous machines, no matter if their $L_{d}$ is equal or not to $L_{q}$. Since the ESCC is capable to control isotropic as well as anisotropic synchronous machines it represents a real extension of the SCC scheme, not only a special-version for anisotropic machines.

\section{Implementation}

\section{Least-Squares-Estimator \& Interpolation Algorithm}

An illustration of the implementation of the ESCC is displayed in figure 4. The currents are measured with a high sampling rate and oversampling during pulse period $\mathrm{k}$, and are input to the least-squaresestimator algorithm. Here the two characteristic values of the switching states that have been applied during period $\mathrm{k}$ are calculated. This is $\Delta \underline{i}_{a, n, k}$ for the active switching state vector to the right of $\underline{i}_{a, k}$ (index n), $\Delta \underline{i}_{a, n+1, k}$ for the one to the left of $\underline{i}_{a, k}$ (index $\mathrm{n}+1$ ) and for the two freewheeling states $\Delta \underline{i}_{f, 1, k}$ and $\Delta i_{f, 2, k}$ (see also fig.1 and fig.2(b)). In addition to that, the value of the stator current vector at the end of the current pulse period $i_{e, k}$ is calculated by linear extrapolation from the measured characteristic values and the knowledge of the applied duty cycles. The characteristic value $\Delta \underline{i}_{f, k}$ can optionally be calculated as the mean value of the two measured values $\Delta \underline{i}_{f, 1, k}$ and $\Delta \underline{i}_{f, 2, k}$ to improve accuracy.

To get sufficiently accurate results from the least squares estimator algorithm, a minimum number of measurement points is necessary, making a high sampling rate essential for the SCC and the ESCC scheme. In the implemented simulation of the ESCC scheme and the existing test bench, that was used to prove the basic SCC scheme [8], a minimum of ten measurement points per switching state was sufficient to get accurate characteristic values. Depending on the implemented sampling rate, this restriction leads to pulse periods in which not all four switching states are long enough, so that the characteristic values can be measured and calculated directly with the least squares estimator algorithm. Nevertheless, those characteristic values can be calculated by linear interpolation between the values of the adjacent current slopes and the knowledge of the applied duty cycles. This is done directly after the least squares estimator algorithm, so that the characteristic values of both applied active switching states and the freewheeling switching states are known from either measurement or interpolation in every pulse period. 


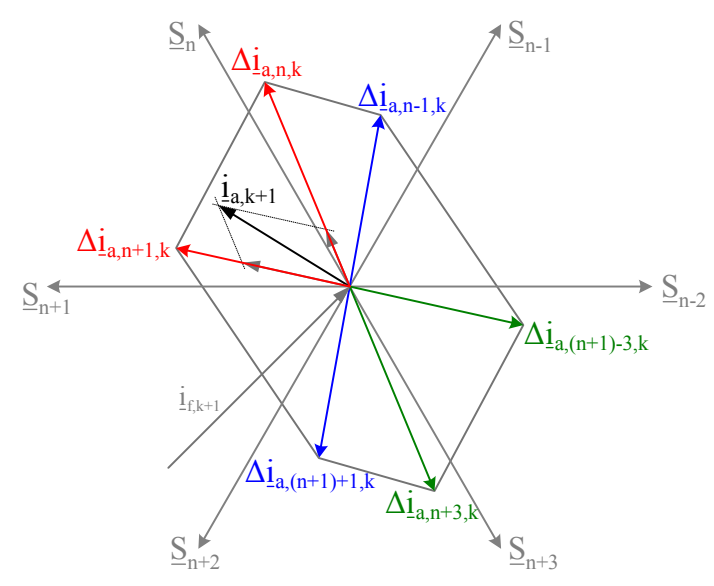

(a) Calculation of all six active characteristic values. Red: measured / interpolated Green: measured values rotated by $180^{\circ}$ Blue: calculated with eq. 24 .

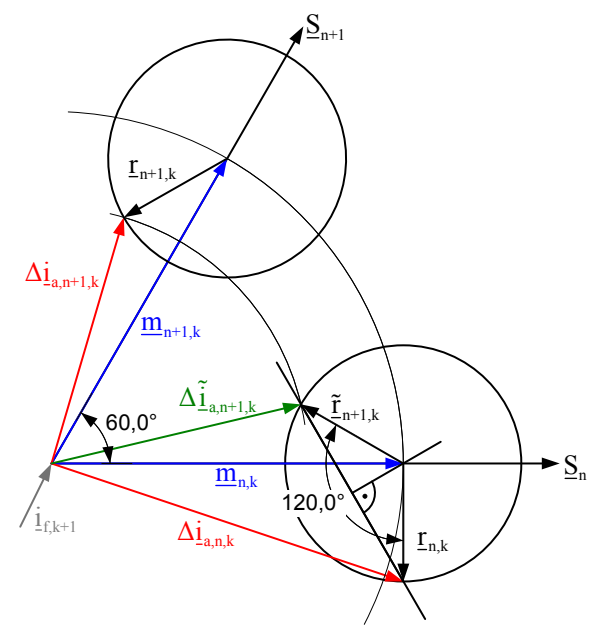

(b) Calculation of $\underline{m}_{n}$ by building the perpendicular bisector of the two measured values $\Delta \mathrm{i}_{\mathrm{a}, \mathrm{n}, \mathrm{k}}$ and $\Delta \tilde{\mathrm{i}}_{\mathrm{a}, \mathrm{n}+1, \mathrm{k}}=\Delta \mathrm{i}_{\mathrm{a}, \mathrm{n}+1, \mathrm{k}} \cdot e^{-j \frac{\pi}{3}}$ and calculating the intersection point with the switching state vector's line

Figure 5: Calculation of all six active characteristic values

\section{Calculation of all six active characteristic values}

The knowledge of all six characteristic values for the active switching states (further briefly referred to as "active characteristic values") is mandatory for magnetic anisotropic machines, because they permanently vary in length and angle and the plane that is spanned by them is no regular hexagon anymore (see fig.2(b)). Since only two of the possible six active switching states are applied during one pulse period, only the two characteristic values $\Delta \underline{i}_{a, n, k}$ and $\Delta \underline{i}_{a, n+1, k}$ can really be measured, respectively interpolated. In figure 5(a) they are displayed as the two red vectors, adjacent to $i_{a, k+1}$. The others are calculated, using equation 16 and the symmetric dependencies coming from this.

As already mentioned above, the characteristic values of opposite switching states show a symmetry with respect to the tip of $\underline{i}_{f, k+1}$ (see fig.2(b)). This means that the opposite characteristic values $\Delta \underline{i}_{a, n \pm 3, k}$ and $\Delta \underline{i}_{a,(n+1) \pm 3, k}$ are derived by simply rotating the corresponding measured characteristic value by $180^{\circ}$.

$$
\begin{aligned}
\Delta \underline{i}_{a, n \pm 3, k} & =\Delta \underline{\underline{i}}_{a, n, k} \cdot e^{j \pi} \\
\Delta \underline{i}_{a,(n+1) \pm 3, k} & =\Delta \underline{i}_{a, n+1, k} \cdot e^{j \pi}
\end{aligned}
$$

The index $n \pm 3$ denotes, that the switching state index $\mathrm{n}$ for the active switching states must remain in the defined range of $n \in\{1 . .6\}$ and that the sign has to be chosen depending on the value of $n$. They are marked in figure $5(\mathrm{a})$ as the green vectors.

The two remaining characteristic values $\Delta \underline{i}_{a, n-1, k}$ and $\Delta \underline{i}_{a,(n+1)+1, k}$ (blue vectors in fig.5(a)) can be obtained by solving equation 16 . Therefore the two vectors $\underline{m}_{n, k}$ and $\underline{r}_{n, k}$ are necessarily required (see eq. 17-20).

The vectors $\underline{m}_{n}$ are constant and the angles $\varphi_{n}$ are known from their corresponding switching state $S_{n}$. The vectors $\underline{r}_{n, k}$ all have the same length and rotate around the tip of the corresponding vector $\underline{m}_{n, k}$, describing circles (3). The length of $\underline{m}_{n}$ can be obtained by making use of the geometric dependencies of the active characteristic values:

The arguments of the six $\underline{r}_{n, k}$ of one pulse period k differ in the angular offset, caused by $\varphi_{n}$. The angle between the vectors $\underline{r}_{n, k}$ and $\underline{r}_{n+1, k}$ of two adjacent switching states is always $-60^{\circ}$ :

$$
\arg \left(\underline{r}_{n+1}\right)-\arg \left(\underline{r}_{n}\right)=2 \gamma(t)-\varphi_{n+1}-2 \gamma(t)+\varphi_{n}=\varphi_{n}-\varphi_{n+1}=-\frac{\pi}{3}
$$

Since the length of all $\underline{m}_{n}$ and $\underline{r}_{n, k}$ is equal, the measured characteristic value $\Delta \underline{i}_{a, n+1, k}$ can be rotated by this angle as displayed in figure 5(b), to generate an artificial intersection point with the circle described 
by $\underline{r}_{n, k}$ around the tip of $\underline{m}_{n, k}$.

$$
\begin{aligned}
\Delta \tilde{\mathrm{i}}_{\mathrm{a}, \mathrm{n}+1, \mathrm{k}} & =\Delta \underline{\mathrm{i}}_{\mathrm{a}, \mathrm{n}+1, \mathrm{k}} \cdot e^{-j \frac{\pi}{3}} \\
\tilde{\mathrm{r}}_{\mathrm{n}+1, \mathrm{k}} & =\underline{\mathrm{r}}_{\mathrm{n}+1, \mathrm{k}} \cdot e^{-j \frac{\pi}{3}}
\end{aligned}
$$

With this intersection point, two points on this circle are given within the same pulse period and the length $m_{n}$ of $m_{n}$ can be calculated instantly within just one pulse period. This is done by building the perpendicular bisector of those two points $\Delta \underline{i}_{a, n, k}$ and $\Delta \tilde{\mathrm{i}}_{a, n+1, k}$ and calculate the intersection point with the switching state vector's line $\underline{S}_{n, \text { axis }}=\underline{i}_{f, k+1}+\infty \cdot e^{j \varphi_{n}}$.

If for example the applied active switching states in period k have been $S_{3}$ and $S_{4}$ as displayed for period $\mathrm{k}+1$ in figure 2(b), then $\Delta \underline{i}_{a, 3, k}$ and $\Delta \underline{i}_{a, 4, k}$ have been measured. $\Delta \underline{i}_{a, 6, k}$ can now be calculated as a vector of the same length and with an argument of that of $\Delta i_{a, 3, k+1}$, rotated by $180^{\circ}$. The calculation of $\Delta i_{a, 1, k}$ is similar:

$$
\begin{aligned}
\Delta \underline{i}_{a, 6, k} & =\Delta \underline{i}_{a, 3, k} \cdot e^{j \pi} \\
\Delta \underline{i}_{a, 1, k} & =\Delta \underline{i}_{-a, 4, k} \cdot e^{j \pi}
\end{aligned}
$$

Once $\underline{m}_{n, k}$ is known, a simple vector subtraction leads to the rotating vector $\underline{r}_{n, k}$ (see equation 16) and the two remaining characteristic values can be obtained by solving equation 16 :

$$
\begin{aligned}
& \Delta \underline{i}_{a, n+1, k}=\underline{m}_{n+1, k}-\underline{r}_{n+1, k}=\underline{m}_{n, k} \cdot e^{j \frac{\pi}{3}}-\underline{r}_{n, k} \cdot e^{-j \frac{\pi}{3}} \\
& \Delta \underline{i}_{a, n-1, k}=\underline{m}_{n-1, k}-\underline{r}_{n-1, k}=\underline{m}_{n, k} \cdot e^{-j \frac{\pi}{3}}-\underline{r}_{n, k} \cdot e^{j \frac{\pi}{3}}
\end{aligned}
$$

Or generally:

$$
\Delta \underline{i}_{a, n \pm w, k}=\underline{m}_{n, k} \cdot e^{ \pm j \frac{\pi}{3} \cdot w}-\underline{r}_{n, k} \cdot e^{\mp j \frac{\pi}{3} \cdot w} \quad w \in\{1 . .6\}
$$

The equations show, that by measuring only the two characteristic values of the adjacent switching states, all six characteristic values can be obtained. That means, that only one pulse period is enough to completely identify the stator current response to all possible switching states and therefore the current control loop system dynamics.

\section{Selection of Switching States \& Calculation of duty-cycles}

In contrary to conventional SVM the segment, and with that the set of two adjacent switching state vectors, can not be changed every $60^{\circ}$ anymore. It has to be decided in every single switching cycle, which of the possible six pairs of adjacent active switching states leads to the desired current change $\underline{i}_{a, k+1}$. This can be done by simply calculating the duty-cycles for all six pairs of adjacent active switching states. The pair, where both duty cycles are greater or equal to zero then is to be taken.

For the calculation of the duty-cycles the desired value $\underline{i}_{a, k+1}$ is necessary (see figure 2(b) and 4).

$$
\underline{i}_{a, k+1}=\underline{i}_{e, k+1}-\underline{i}_{f, k+1}=\underline{i}_{e, k+1}-i_{e, k}-\Delta i_{f, k}
$$

The calculation of the duty cycles itself is done with the known techniques also applied in conventional SVM. The results of these calculations are the duty cycles $d_{n, k+1}=\frac{T_{a, n, k+1}}{T_{p}}$ for the active switching state corresponding to the characteristic value $\Delta \underline{i}_{a, n, k+1}, d_{n+1, k+1}=\frac{T_{a, n+1, k+1}}{T_{p}}$ for the one corresponding to $\Delta \underline{i}_{a, n+1, k+1}$ and $f=\frac{T_{F 1}+T_{F 2}}{T_{p}}$ for the two freewheeling states. Those duty cycles are input to the space vector modulator, which uses them to output the gate signals according to the implemented pulse pattern.

\section{Sensorless control}

The vectors $\underline{r}_{n}$ in equation 16 obviously show a dependency on the rotor position angle $\gamma$ (see equation 16 and 18). Since the measuring of those vectors is inherent in the proposed control scheme, it is particularly suitable for sensorless control. It is possible to gain the rotor position angle at no extra cost over the whole speed range, including low speed and standstill. Since many years the INFORM-Method is a 
known technique that makes use of the same effect in synchronous machines with $L_{d} \neq L_{q}[10,3,5]$. The calculation that has to be done to get the rotor-position angle from the vector $\underline{r}_{n}$ is:

$$
\gamma=\frac{\arg \left(\underline{r}_{n}\right)+\varphi_{n}}{2}=\frac{1}{2}\left(\arctan \left(\frac{m_{n, \beta}-\Delta i_{a, n, \beta}}{m_{n, \alpha}-\Delta i_{a, n, \alpha}}\right)+\varphi_{n}\right)
$$

Since the argument of $\underline{r}_{n}$ is proportional to $2 \gamma$, the result of this equation has no information, whether this is $e^{j \gamma}$ or $e^{-j \gamma}$, that points in the direction of the d-axis. One possibility to solve this issue is to consider the characteristic value of the freewheeling switching states and run an observer. The condition that must be met to operate the ESCC sensorless, is that there is a strong inequality in $L_{d}$ and $L_{q}$. The more the machines' $L_{d}$ and $L_{q}$ are similar, the shorter the vectors $\underline{r}_{n}$ will be, which means that at some point, the measurement of the angle of $\underline{r}_{n}$ will be impracticable.

\section{Simulation results}

The presented ESCC control scheme has been implemented in a Matlab/Simulink-simulation to develop and proof the theory. The simulation parameters have been taken from the hardware test plant, that was used by [8] to proof the SCC for synchronous machines with $L_{d}=L_{q}$. The main simulation parameters are listed in table I.

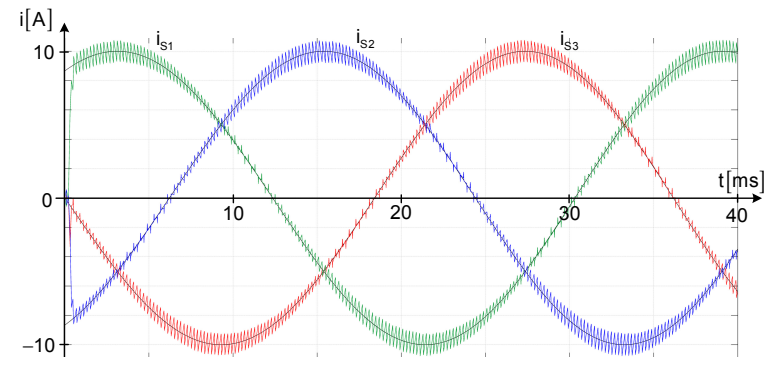

(a) Startup and steady state operation $\left(L_{d} \neq L_{q}\right)$

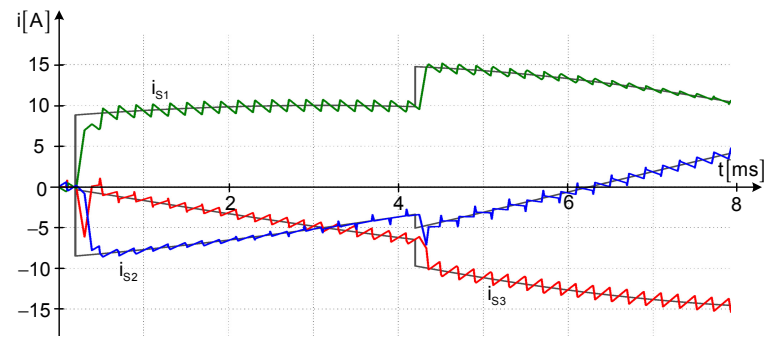

(c) Startup and setpoint step of 5A in $i_{q}\left(L_{d} \neq L_{q}\right)$

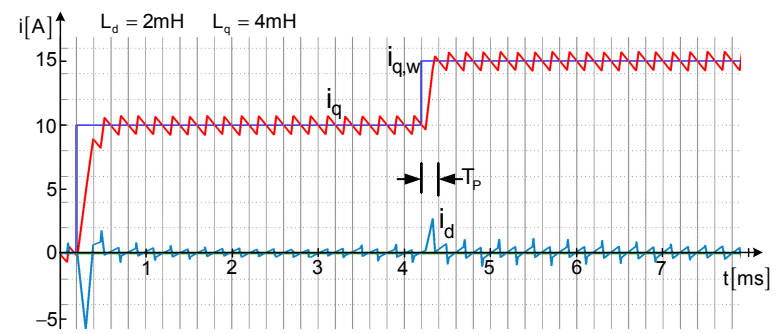

(e) Startup and setpoint step of 5A in $i_{q}$ in the rotor oriented reference frame $\left(L_{d} \neq L_{q}\right)$

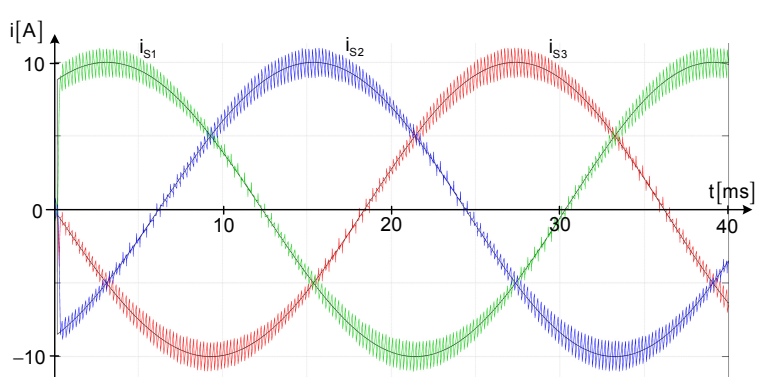

(b) Startup and steady state operation $\left(L_{d}=L_{q}\right)$

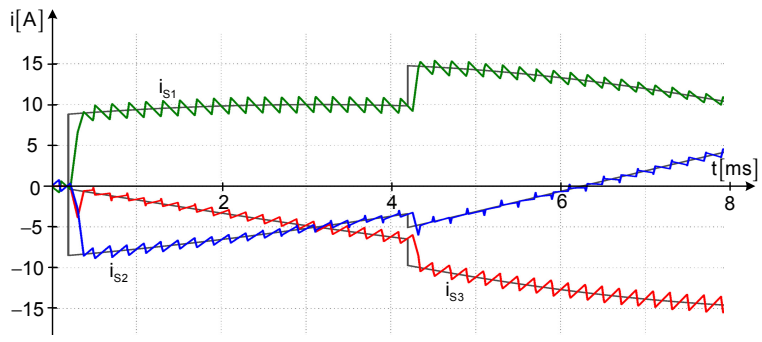

(d) Startup and setpoint step of 5A in $i_{q}\left(L_{d}=L_{q}\right)$

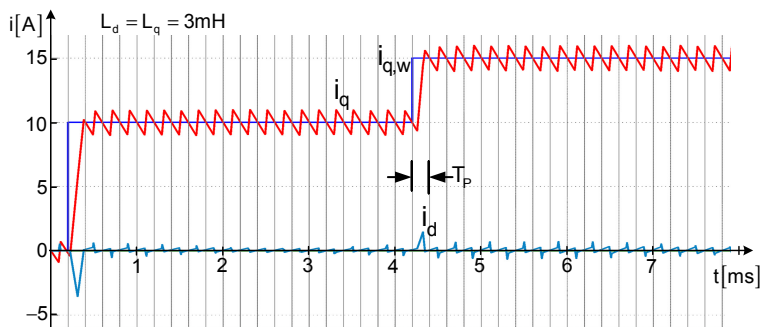

(f) Startup and setpoint step of 5A in $i_{q}$ in the rotor oriented reference frame $\left(L_{d}=L_{q}\right)$

Figure 6: Simulation of startup, steady state operation and setpoint step response with the extended straightforward current control scheme presented in this paper for a magnetic anisotropic machine with $L_{d}=2 \mathrm{mH}$ and $L_{q}=4 \mathrm{mH}$ (figures 6(a), 6(c)), 6(e)) and a magnetic isotropic permanent magnet synchronous machine with $L_{d}=L_{q}=3 \mathrm{mH}$ (figures 6(b), 6(d)), 6(f)) at $n=400 \mathrm{~min}^{-1}, \mathrm{p}=4, f_{p}=5 \mathrm{kHz}, i_{d, w}=0 \mathrm{~A}$. 
Table I: main simulation parameters

\begin{tabular}{lcc}
\hline Parameter & anisotropic & isotropic \\
\hline DC-link Voltage $U_{D C}$ & $400 \mathrm{~V}$ & $400 \mathrm{~V}$ \\
Inductance $L_{d}$ & $2 \mathrm{mH}$ & $3 \mathrm{mH}$ \\
Inductance $L_{q}$ & $4 \mathrm{mH}$ & $3 \mathrm{mH}$ \\
Pulse Period $T_{p}$ & $200 \mu \mathrm{s}$ & $200 \mu \mathrm{s}$ \\
Sample Rate $T_{A D}$ & $0.8 \mu \mathrm{s}$ & $0.8 \mu \mathrm{s}$ \\
\hline
\end{tabular}

The figures 6(a) and 6(b) show the stator currents with the presented ESCC algorithm in startup and steady state condition. A closer look at startup and a setpoint step of 5A in $i_{q}$ is displayed in 6(c) and 6(d). This setpoint step is also shown in the rotor-oriented reference frame with $i_{d}$ and $i_{q}$ in 6(e) and 6(f). The excellent dynamics and steady state accuracy is obvious and shows that the ESCC is well suited to control isotropic or anisotropic machines without a change in the algorithm.

\section{Conclusion}

This paper describes how the Straightforward Current Control scheme presented in $[6,7,8]$ has to be modified to be able to also control magnetic anisotropic machines. The necessary equations are derived from the system equations of the permanent magnet synchronous machine and the control scheme is presented. The rotor position angle can be obtained directly from the inner control variables, inherent in the proposed ESCC control scheme. The control quality and the dynamics are demonstrated with simulation results.

\section{References}

[1] P. Cortes, M. Kazmierkowski, R. Kennel, D. Quevedo, and J. Rodriguez, "Predictive control in power electronics and drives," Industrial Electronics, IEEE Transactions on, vol. 55, no. 12, pp. $4312-4324$, dec. 2008.

[2] A. Linder and R. Kennel, "Model predictive control for electrical drives," in Power Electronics Specialists Conference, 2005. PESC'05. IEEE 36th, june 2005, pp. 1793 -1799.

[3] M. Schrödl, "Sensorless control of ac machines at low speed and standstill based on the informmethod," in Industry Applications Conference, 1996. Thirty-First IAS Annual Meeting, IAS '96., Conference Record of the 1996 IEEE, vol. 1, oct 1996, pp. 270 -277 vol.1.

[4] P. Landsmann, C. Hackl, and R. Kennel, "Eliminating all machine parameters in encoderless predictive torque control without signal injection," in Electric Machines Drives Conference (IEMDC), 2011 IEEE International, may 2011, pp. 1259 -1264.

[5] M. Schrödl and C. Simetzberger, "Sensorless control of pm synchronous motors using a predictive current controller with integrated inform and emf evaluation," in Power Electronics and Motion Control Conference, 2008. EPE-PEMC 2008. 13th, sept. 2008, pp. 2275 -2282.

[6] J. Weigold and M. Braun, "Predictive current control using identification of current ripple," IEEE Transactions on Industrial Electronics, vol. 55, no. 2, pp. 4346-4353, 2008.

[7] F. Becker, H. Ennadifi, and M. Braun, "Straightforward current control - one step controller based on current slope detection," in EPE ECCE-EUROPE 2011 Birmingham, 2011.

[8] F. Becker, "Ein neues adaptives verfahren zur hochdynamischen stromregelung," Ph.D. dissertation, Elektrotechnisches Institut, Karlsruher Institut für Technologie (KIT), 2011.

[9] H. Späth, Elektrische Maschinen - Eine Einführung in die Theorie des Betriebsverhaltens. Springer-Verlag, 1973.

[10] M. Schrödl, "Sensorless control of permanent magnet synchronous motors," Electric Machines \& Power Systems, vol. 22, no. 2, pp. 173-185, 1994. 